\title{
Sistemas neurales de relevancia en el contexto de la psicobiología de la ansiedad: una revisión
}

\author{
Alberto Morales, Carmen Torres y Jesús L. Megías
}

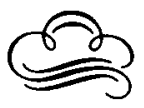

Resumen

La ansiedad es un estado emocional central de características muy complejas que, al menos en términos de respuesta, se asemeja en gran medida al miedo. En las últimas décadas, estudios neurobiológi$\cos y$ psicobiológicos ban identificado numerosas estructuras y circuitos cerebrales estrechamente relacionados con este tipo de respuestas, asi como con el estado central característico. Destacan, por su especial relevancia, el sistema límbico (incluyendo el sistema septobipocampal, la amígdala y el bi potálamo posterior) y los sistemas monoaminérgicos ascendentes (principalmente de serotonina y norepinefrina), lugares donde, además, las sustancias ansiolíticas podrian ejercer su efecto terapéutico. El principal objetivo del presente trabajo es revisar la precisa implicación de estos sistemas neurales en la modulación de ese estado emocional definido como ansiedad.

Palabras clave: Amígdala, Ansiedad, Ansiolíticos, Hipotálamo Posterior, Sistema Límbico, Sistema Septohipocampal, Sistemas Monoaminérgicos ascendentes.

\section{Abstract}

Anxiety is a complex, central emotional state which shares common characteristics with fear. Neurobiological and psychobiological investigations have identified, during the last decades, several brain structures and circuits closely related to the above-mentioned central state. Of special importance are the limbic system (including the septohippocampal system, the amygdaloid complex, and the posterior bypothalamus) on which the anxiolytic drugs could exert their therapeutic action. This paper reviews the precise involvement of these neural systems in the control of the emotional state we call anxiety.

Key words: Amygdala, Anxiety, Anxiolytics, Ascending monoaminergic system; Limbic system, Posterior hypothalamus, Septohippocampal system.

Dirección de los autores: Departamento de Psicología Experimental y Filosofía del Comportamiento. Universidad de Granada 
La definición del término ansiedad, de forma similar a lo ocurrido en otras categorías psicopatológicas, no ha estado exenta de controversias y sólo recientemente se ha alcanzado un cierto consenso unificador en torno a los criterios definitorios propuestos por la Sociedad Americana de Psiquiatría (American Psychiatric Association, 1984). Este planteamiento se caracteriza por un marcado énfasis psiquiátrico y taxonómico, en contraste (que no en oposición) con otros de matiz quizás más psicológicos, para los que la ansiedad podría definirse como «un patrón o conjunto complejo y variable de conductas, caracterizado por sentimientos subjetivos de aprensión y tensión emocional, acompañado o asociado a una intensa activación fisiológica y a determinados patrones de conducta manifiesta, que tiene lugar más o menos característicamente en respuesta a determinados estímulos, tanto internos como externos, del medio en el que se desenvuelve el sujeto» (Carrobles, 1989). En cualquier caso, y al margen de esta polémica, sí existe un común acuerdo: la ansiedad es un estado emocional de características muy peculiares que, en términos de respuesta, se asemeja en gran medida al miedo. En este último caso es posible identificar con claridad un estímulo amenazador, que supone un peligro para la integridad física del sujeto; en el caso de la ansiedad, el estímulo desencadenante es mucho más difuso y ambiguo, y, en ocasiones, difícil de identificar. Ante esta situación, en individuo manifiesta una sensación de desagrado y malestar característico, incluyendo síntomas que oscilan desde ligeras palpitaciones y sudoración hasta pérdida de sueño y sensación de muerte inminente (Ayuso, 1988). En un grado adecuado, esta reacción posee un evidente valor adaptativo, ya que supone la preparación del sujeto para enfrentarse a situaciones potencialmente peligrosas. No obstante, y como se desprende de los numerosos datos clínicos existentes, cuando dicha reacción es desproporcionadamente intensa o persiste durante un tiempo excesivo, puede devenir en un estado patológico (ansiedad patológica, en sus múltiples manifestaciones) gravemente perturbador de la conducta (Clare, 1985).

A pesar de las dificultades definitorias arriba esbozadas, la ansiedad es, sin duda, el estado emocional que en mayor medida ha atraído la atención de los investigadores interesados en el estudio del sustrato biológico de la conducta emocional. En este sentido, es obligatorio referirse a los trabajos pioneros de Papez, quien delineó y sistematizó por vez primera (si bien de forma más teórica que experimental) un circuito cerebral directamente involucrado en el control de la emoción, que incluía estructuras prosencefálicas de localización límbica: hipotálamo, giro del cíngulo, formación del hipocampo y regiones talámicas de proyección cingular (Brodal, 1981). Actualmente se sabe que, en efecto, este circuito (conocido como «circuito de Papez») es central en el control de las emociones y, por ende, de la ansiedad. En la actualidad ha sido caracterizado con mayor precisión, incluyendo otras estructuras de localización asimismo límbicas, como la amígdala y el área septal (Nauta y Feirtag, 1987).

En el trabajo que a continuación se desarrolla, se revisarán las principales estructuras y circuitos cerebrales implicados en el control de la ansiedad, y que han sido delineados merced al empleo de numerosas técnicas, tanto clínicas como experimentales. Esta metodología ha generado una considerable cantidad de resultados, provenientes, en su mayoría, de las siguientes estrategias de investigación:

1. Empleo de modelos animales de ansiedad, ampliamente aceptados por su buen valor predictivo de, por ejemplo, el carácter ansiolítico o ansiógeno de una determinada sustancia química; entre ellos destacan, por su uso generaliza- 


\section{7}

do, el paradigma de Vogel-Geller y el modelo de los dos compartimentos (Treit, 1985).

2. Estudio de los cambios comportamentales inducidos mediante técnicas de lesión cerebral (psicocirugía en el caso de sujetos humanos) (Brodal, 1981; Kuhar, 1986).

3. Estudio de los mecanismos de acción y lugares centrales de actuación de las principales sustancias ansiolíticas (Gray, 1982a; Costa, 1985; Iversen, 1985).

4. Empleo de técnicas de análisis del tejido cerebral in vivo, en sujetos humanos conscientes, destacando la tomografía por emisión de positrones (PET), que permite no sólo una visualización estructural del cerebro, sino también funcional (Trimble, 1986).

Como ya se ha señalado, las investigaciones de Papez centraron la atención en el sistema límbico como posible sustrato biológico de la conducta emocional. El sistema límbico agrupa a numerosas estructuras cerebrales de localización diencéfalo-prosencefálica, y fue descrito por vez primera por Broca en la segunda mitad del siglo XIX, para referirse aquellas regiones nerviosas ubicadas en la base de los hemisferios cerebrales y en proximidad al «borde» (limbus) de la corteza cerebral. En la actualidad se considera que el sistema límbico incluye estructuras tanto corticales como subcorticales, estrecha y complejamente interconectadas, entre las que destacan (Nauta y Feirtag, 1987; Lopes da Silva, 1990):

- La corteza límbica, que incluye regiones cerebrales filogenéticamente antiguas como, por ejemplo, la paleocorteza y la arquicorteza. Esta última comprende estructuras nerviosas de gran importancia en el presente contexto, como formación del hipocampo (giro dentado, hipocampo y subículum), pre, post y parasubículum, corteza entorrinal y circunvolución del cíngulo.

- El complejo nuclear amigdalino, con tres grandes subdivisiones: corticomedial, central y basolateral.

- El área septal, principalmente en su división media.

- El núcleo accumbens, de localización asimismo prosencefálica, en la proximidad de la comisura anterior.

- Determinadas estructuras hipotalámicas, como el área preóptica y, sobre todo, la región hipotalámica posterior y los cuerpos mamilares.

- El complejo nuclear talámico-anterior.

Aunque estas estructuras cerebrales están implicadas en el control de numerosos aspectos de la conducta, la emoción es, sin duda, uno de los más importantes. En este trabajo se revisarán las áreas límbicas de importancia en la mediación de la ansiedad, así como otros sistemas relacionados con ellas, que, sin tener una localización límbica, también están involucrados en su control.

\section{EL SISTEMA SEPTOHIPOCAMPAL}

El área septal y la formación del hipocampo son estructuras anatómicas de enorme complejidad, cuyas funciones han sido extensamente estudiadas en el contexto de la investigación animal. En este sentido destaca el intento unificador teórico-experimental realizado por J. Gray, para quien el sistema septohipocampal se relaciona primariamente con el control de las respuestas de un organismo tradicionalmente asociadas a la ansiedad (Gray 1982a, b). Los presupuestos básicos que sustentan este argumento son los siguientes: 


\section{8}

- Existe una estrecha similitud entre los efectos comportamentales inducidos en animales por las sustancias ansiolíticas (principalmente benzodiacepinas, barbitúricos y etanol) y aquellos derivados de lesiones septales e hipocámpicas (Gray, 1977).

- Los circuitos intrahipocámpicos son especialmente sensibles a los cambios contextuales producidos en el ambiente. Así, las células de la región CA3 (principalmente de tipo piramidal) muestran una clara habituación de respuesta ante la presentación repetitiva de estímulos irrelevantes o inocuos (Vinogradova, 1975); por el contrario, si el estímulo es de naturaleza aversiva, biológica o adquirida por condicionamiento, las citadas células no sólo no habitúan su respuesta sino que la incrementan significativamente (Thompson y colbs., 1980). Este efecto está mediado, a su vez, por los sistemas monoaminérgicos ascendentes que alcanzan el sistema septohipocampal (Segal, 1980). Asimismo, el ritmo «theta», originado en el área septal medial y que se transmite a las células del hipocampo a través de la vía septohipocampal (de naturaleza colinérgica), parece modular el paso de información biológicamente relevante a través de los circuitos intrahipocámpicos anteriormente referidos (Gray, 1982a).

El sistema septohipocampal, y en especial la región subicular, funcionaría a modo de comparador entre las expectativas del organismo (lo que, teóricamente, se llevaría a cabo a través de circuito de Papez) y la realidad externa (que llegaría al hipocampo a través de las vías perforantes entorrinales, como información multimodal altamente procesada). Cuando se produjese un desajuste entre ambas («mismatch»), y una vez detectado en el subículum, el sistema septohipocampal asumiría el control de la conducta, generando, entre otras manifestaciones, inhibición del comportamiento e incremento en los niveles de activación y atención. En un contexto experimental, Gray ha identificado algunas situaciones inductoras del citado desajuste y, por ende, productoras de ansiedad, como: I: estímulos novedosos, II: estímulos asociados al castigo, III: estímulos inductores de miedo incondicionado y IV: disminución en la tasa de recompensa («frustrative non-reward») (Gray, 1982a).

El modelo propuesto por Gray, aunque sumamente especulativo en alguno de sus planteamientos (ver especialmente Gray, 1982b), ha tenido un amplio eco en la comunidad científica aneja al tema y, ciertamente, cuenta con un decisivo apoyo experimental. No obstante, y asimismo de acuerdo con Gray, el sistema setohipocampal sería sólo una parte de los circuitos centrales implicados en el control de la ansiedad, aunque de especial relevancia.

En sujetos humanos, la región parahipocampal, incluyendo el subículum, parece, efectivamente, estar involucrada en la ansiedad. Así, en un estudio donde se midió la tasa de flujo sanguíneo cerebral (mediante PET) en sujetos afectos de crisis de pánico, se comprobó la existencia de una clara anomalía en la citada región cerebral, principalmente del hemisferio izquierdo; dicha anomalía no se observó en sujetos normales ni en aquellos otros que, aun padeciendo crisis de pánico, éstas no eran químicamente inducibles (mediante la administración sistemática de lactato, por ejemplo) (Reiman y colbs., 1984). En un estudio posterior se comprobó, y haciendo uso de la misma técnica, cómo el flujo sanguíneo cerebral se incrementaba sensiblemente en la región parahipocampal de sujetos normales a quienes se presentaba una señal indicadora de la inminente administración de un choque eléctrico. La región parahipocampal, por tanto, parecía relacionarse con un estado central indicativo de ansiedad anticipatoria (Reiman y colbs., 1989). 


\section{9}

\section{EL COMPLEJO NUCLEAR AMIGDALINO}

De acuerdo con la extensa literatura disponible, la amígdala parece ser un centro clave en el control de la expresión emocional, ya que, y fundamentalmente a través de sus conexiones eferentes, modula respuestas de naturaleza endocrina, autonómica y somatomotora relacionadas con los estados emocionales (Brodal, 1981; Sarter y Markowitch, 1985)

La amígdala, o, más propiamente hablando, el complejo nuclear amigdalino, es una voluminosa estructura cerebral inmersa en el lóbulo temporal y localizada en posición anterior a la formación del hipocampo. Se subdivide en varios grupos nucleares, siendo los más importantes el basolateral, el central y el corticomedial (Carpenter, 1978). Concretamente la región central ha recibido especial atención, ya que establece conexiones con regiones implicadas en el control de conductas típicamente asociadas a situaciones de miedo (Davis, 1989).

$\mathrm{Al}$ igual que el sistema septohipocampal, la amígdala ha sido extensamente estudiada en un contexto tanto experimental (especies no humanas) como clínico (sujetos humanos), derivándose su clara implicación en las respuestas de ansiedad. Así, y a modo de ejemplo, podrían citarse las siguientes investigaciones:

- La lesión del núcleo central de la amígdala en ratas abole el reflejo de sobresalto condicionado («fear-potentiated startle paradigm»), respuesta conductual potencialmente indicativa de un estado central de ansiedad. Idéntico efecto se consigue mediante la administración intraamigdalina de diacepam. Por el contrario, y como era de esperar, la estimulación eléctrica del núcleo central de la amígdala produce un claro incremento en la respuesta refleja anteriormente comentada (Davis, 1989).

- La administración central de benzodiacepinas en regiones de la amígdala ricas en receptores para estas sustancias (Niehof y Kuhar, 1983), induce un mayor número de respuestas castigadas en el paradigma de conflicto, lo que sugiere un efecto ansiolítico por parte de las mismas (Scheel-Krüger y Petersen, 1982; Shibata y colbs., 1982; Kataoka y colbs., 1987).

- La actividad eléctrica del núcleo central de la amígdala se ve modificada ante la presencia de estímulos condicionados de naturaleza aversiva (adquirida ésta mediante un proceso de condicionamiento pavloviano) (Pascoe y Kapp, 1985).

En resumen, la amígdala parece desempeñar un papel importante en la conducta emocional, en especial en aquellas situaciones asociadas a miedo/frustración, de naturaleza condicionada o incondicionada, y de relevancia en una situación de ansiedad. A través del sistema amigdalino se modularían las respuestas periféricas que caracterizan los estados de naturaleza aversiva en general, y ansiosos en particular.

La literatura concerniente al papel de la amígdala en la conducta humana es también rica y, en general, complementaria a la obtenida en experimentación animal. En efecto, en fechas muy cercanas a la propuesta del circuito de Papez como clave en el procesamiento de la conducta emocional, Klüver y Bucy publicaron su ya clásico estudio sobre el efecto comportamental de lesiones del lóbulo temporal en primates. Estas lesiones inducían, entre otras cosas, lo siguiente: tendencias orales, entendidas como un examen de los objetos a través de la boca, incremento en la actividad sexual, tendencia a prestar atención a un gran número de estímulos visuales, «aplanamiento» de la conducta emocional $\mathrm{y}$, a veces, ausencia de la misma. Estos síntomas, agrupados bajo la denominación de síndrome de Klüver-Bucy, parecen relacionarse con el daño infligido a la amíg- 
dala, dato que ha sido refrendado a partir de estudios realizados en sujetos humanos sometidos a ablación de ésta con fines terapéuticos (Brodal, 1981). Más aún, la estimulación eléctrica de la amígdala en humanos conscientes se asocia a intensas sensaciones de miedo y ansiedad (Chapman y colbs., 1954), en concordancia asimismo con lo observado en sujetos aquejados de ataques psicomotores, en los que el foco epiléptico se sitúa en o próximo al sistema amigdalino (Brodal, 1981; Smith, et al., 1989; Meldrun, 1990).

\section{LOS SISTEMAS MONOAMINERGICOS ASCENDENTES}

Los sistemas monoaminérgicos se originan, fundamentalmente, en agrupaciones nucleares de localización troncoencefálica, inervando amplias zonas del Sistema Nervioso Central a través de vías ascendentes y descendentes. Serotonina (5-HT) y norepinefrina (NE) han recibido especial atención en el estudio de la ansiedad, ya que, como se verá, las sustancias ansiolíticas podrían ejercer su efecto terapéutico a través de dichos sistemas (Iversen, 1985).

La 5-HT es una sustancia química perteneciente al grupo de las indolaminas y localizada a nivel central en el sistema del rafé, conjunto de núcleos troncoencefálicos de los que tres de ellos se ubican en el mesencéfalo. De especial relevancia son el rafé medial (B8) y el rafé dorsal (B7), cuyas eferencias alcanzan importantes regiones límbicas a través del fascículo prosencefálico medial (Steinbusch y Nieuwenhuys, 1983).

Aunque no en su totalidad, la mayoría de los datos experimentales obtenidos sugieren que un descenso en los niveles centrales de 5-HT tiene efectos ansiolíticos (Chopin y Briley, 1987; Kahn y colbs., 1988). Las benzodiacepinas y otras sustancias ansiolíticas podrían producir este efecto inhibiendo al sistema serotonérgico mesencefálico. En este sentido, ha podido observarse lo siguiente:

- Los núcleos serotonérgicos mesencefálicos poseen receptores benzodiacepínicos (Gallager, 1978; Fonnum, 1987) e inervan estructuras límbicas tradicionalmente implicadas en el control de la ansiedad, como, por ejemplo, el hipocampo (Steinbusch y Nieuwenhuys, 1983).

- La lesión química (con la neurotoxina 5,7-dihidroxitriptamina) del sistema serotonérgico ascendente se acompaña de signos comportamentales indicativos de ansiolisis, como, por ejemplo, un decremento en la tasa de supresión condicionada (la conducta parece quedar, pues, «desinhibida») (Iversen, 1985). Estos efectos comportamentales pueden observarse, asimismo, como resultado de la administración de fármacos ansiolíticos y sustancias que reducen los niveles funcionales de 5-HT (Eison y colbs., 1986).

- Las benzodiacepinas inducen un decremento en los niveles límbicos de 5-HT, efecto mediado a través de una inhibición de las células del rafé mesencefálico (Iversen, 1985), o bien directamente sobre las terminales serotonérgicas (Johnston y File, 1986; Nishikawa y Scatton, 1986; Thiebot, 1986; Kahn y colbs., 1988).

- Numerosos modelos animales sugieren un efecto ansiolítico de sustancias serotonérgicas, como, por ejemplo, la buspirona. Así, la administración intrahipocampal y sistemática de esta sustancia produce un efecto anticonflicto (Taylor y colbs., 1985), incrementa la conducta exploratoria en un laberinto elevado (Kostowski y colbs., 1989) y afecta negativamente a una tarea de evitación 
pasiva (Rowan y colbs., 1990), aunque se han publicado datos adicionales en los que no se han obtenido resultados concluyentes a este respecto (Gardner, 1986; Pellow y File, 1986; Flaherty, 1990). El mecanismo de acción de la buspirona no es del todo conocido, si bien se sabe de su afinidad por los receptores $5-\mathrm{HT}_{1 \mathrm{~A}}$, lugar donde podría actuar a modo de agonista/agonista parcial (VanderMaelen y colbs., 1986; Traber y Glaser, 1987; O'Connor y colbs., 1990).

Estos datos sugieren, por consiguiente, un claro papal de la 5-HT en el control de las respuestas del organismo en situaciones aversivas. Así, se piensa, y de acuerdo a lo propuesto, entre otros, por Gray (1982a), que los sistemas serotonérgicos ascendentes serían los responsables de la inhibición comportamental observada en un sujeto sometido a una situación potencialmente ansiógena. Las sustancias ansiolíticas, en especial las benzodiacepinas, liberarían al organismo de esta inhibición mediante su actuación a través del sistema rafé (Aghajanian y colbs., 1987; Taylor y colbs., 1985), induciendo un descenso en los niveles de 5-HT en las regiones límbicas inervadas; no obstante, es preciso tener en cuenta otras investigaciones de las que se desprende una actuación directa de estos fármacos sobre el hipocampo (modificando los niveles de 5-HT) y no tanto a través de los núcleos del rafé (Nishikawa y Scatton, 1986; O'Connor, Rowan y Anwyl, 1990). Otras sustancias ansiolíticas como la buspirona, de amplio empleo en clínica humana (Goa y Ward, 1986), podrían ejercer su efecto terapéutico actuando directamente sobre la 5-HT, no descartándose, además, la implicación de sistemas neuroquímicos diferentes (Hjorth y Carlsson, 1982).

El otro sistema monoaminérgico implicado en la ansiedad es el de NE, principalmente a través del fascículo noradrenérgico dorsal ascendente (FNDA). Esta vía se origina en el locus coeruleus, agrupación nuclear de localización pontina y cuyas eferencias alcanzan prácticamente la totalidad del neuroeje. El FNDA inerva, entre otras regiones, el sistema límbico y la corteza cerebral (Moore, 1982).

De forma similar a lo descrito para las vías serotonérgicas ascendentes, una amplia evidencia experimental sugiere la posible modulación de las respuestas de ansiedad por parte del FNDA. Esta evidencia se ha obtenido, sobre todo, a partir de estudios realizados en animales de experimentación, siendo los más representativos los siguientes:

- Fibras noradrenérgicas procedentes del FNDA inervan amplias zonas de la formación del hipocampo, sugiriendo detallados estudios neurofisiológicos su implicación en la modulación del paso de información nerviosa a través de la citada estructura (Segal, 1980; Nicoll y colbs., 1987). Esto es de suma importancia, ya que en presencia de NE, algunas regiones hipocámpicas, como el subículum, incrementan su capacidad analítica en favor de aquella información neural de procedencia entorrinal y de relevancia biológica para el organismo, como sucede en una situación aversiva de carácter ansiógeno (Gray, 1982a).

- La activación del FNDA produce el ajuste de la frecuencia del ritmo theta hipocámpico a valores próximos a los $7,7 \mathrm{~Hz}$, característico de situaciones asociadas a estrés, viéndose este efecto interferido por lesiones químicas (con la neurotoxina 6-hidroxidopamina) del citado fascículo así como por la administración de sustancias ansiolíticas (Gray, 1982a).

- La estimulación eléctrica del locus coeruleus, y por ende la activación del FNDA, induce en primates una respuesta característica de miedo: dilatación pupilar, piloerección, intento de escape de la situación experimental, movimientos mandibulares, arañado, descarga simpática, estado general de alerta, etc. Este patrón comportamental es muy similar al que se observa en circunstan- 


\section{2}

cias naturales donde la ansiedad y el miedo están presentes. Por el contrario, la lesión del locus coeruleus genera un déficit en aquellas respuestas del organismo asociadas a situaciones de amenaza, haciendo al sujeto, además, menos reactivo al estrés (Redmond, 1987).

- Los efectos comportamentales descritos en el punto anterior son, asimismo, susceptibles de manipulación con sustancias psicoactivas estrechamente relacionadas con el sistema noradrenérgico. Así, la administración de clonidina (fármaco agonista por el receptor $\alpha_{2}$ presináptico y que, en consecuencia, disminuye la secreción de NE) reduce la magnitud de las respuestas asociadas al estrés y/o situaciones amenazantes (Eriksson, 1987). En humanos, su aplicabilidad se refleja en el control del síndrome de abstinencia opiáceo, situación vivenciada de forma muy aversiva y amenazante por el sujeto drogodependiente (Gold, 1978a, b). Por el contrario, la yohimbina (fármaco antagonista por el receptor $\alpha_{2}$ presináptico y que, en consecuencia, facilita la liberación de NE) induce justo los efectos opuestos, lo que, en humanos, se traduce como una clara respuesta de ansiedad y, a veces, la aparición de un ataque de pánico (Lader y Bruce, 1986). Los efectos comportamentales asociados a la clonidina y la yohimbina se relacionan estrechamente con una posible actuación directa de éstos sobre el sistema del locus coeruleus (Eriksson, 1987; Redmond, 1987).

- Por otro lado, y en el contexto de la práctica clínica, ha podido comprobarse que el empleo de beta-bloqueadores adrenérgicos alivia los síntomas de ansiedad, principalmente en pacientes con fobia social (Levin y Liebowitz, 1987).

En resumen, y de acuerdo a la evidencia experimental comentada, el sistema noradrenérgico ascendente podría actuar a modo de alarma ante la presentación de estímulos aversivos y amenazantes para el sujeto, generando un estado de alerta en el mismo mediante un incremento general en el nivel de arousal y una activación del tono simpático. Las fibras noradrenérgicas ascendentes originadas en el locus coeruleus podrían ser las responsables del primer efecto, en especial aquellas que inervan regiones límbicas (hipocampo y amígdala) y neocorticales; las vías descendentes hacia los centros simpáticos de ubicación medular modularían el segundo efecto, siendo responsables de algunas de las manifestaciones externas del estado central de ansiedad (es preciso señalar, asimismo, que el sistema simpático ejerce un efecto directo sobre la actividad eléctrica de las células del locus coeruleus, lo que sugiere una influencia recíproca entre ambos, que alcanza su máxima expresión en el contexto de situaciones de especial relevancia biológica para el organismo; Svensson, 1987). Las sustancias ansiolíticas ejercerían su acción terapéutica (al menos en parte) interfiriendo con la activación de este sistema de alarma, lo que en el caso particular de benzodiacepinas y barbitúricos se apoya en la existencia de receptores GABA-A en los cuerpos celulares de las neuronas del locus coeruleus (Gray, 1987).

En conclusión, serotina y norepinefrina, principalmente a través de sus sistemas ascendentes de proyección prosencefálica, modularían dos aspectos claves en una situación de naturaleza potencialmente ansiógena. El primero de ellos analizaría el carácter apetitivo/aversivo de la estimulación aferente, generando, en su caso, inhibición del comportamiento; el segundo actuaría como un sistema de alarma, induciendo un incremento en el estado de arousal y una activación del sistema simpático acorde con la situación externa y/o interna. Las sustancias ansiolíticas liberarían al organismo de la sensación de ansiedad mediante una actuación directa sobre estos sistemas, directa sobre sus lugares de proyección, o sobre ambos. 


\section{LA REGION HIPOTALAMICA POSTERIOR}

La última región cerebral que se analizará en la presente revisión comprende una zona del circuito de Papez que incluye la región del hipotálamo posterior (principalmente el núcleo posterior del hipotálamo y los cuerpos mamilares), y que ha sido, asimismo, relacionada con el control de las respuestas de ansiedad. En efecto, se sabe desde hace tiempo que la lesión quirúrgica de esta región en sujetos humanos (con fines terapéuticos) produce un descenso manifiesto en la ansiedad, lo que ha dado pie a denominar a esta intervención central como «cirugía sedante» (Kuhar, 1986); su activación eléctrica, como paso previo a la lesión, inducía sensaciones de ansiedad que, además, se acompañaban de signos indicativos de activación simpática (Brodal, 1981; Kuhar, 1986).

Los resultados arriba descritos han sido confirmados en experimentación animal, sugiriendo, además, la implicación del sistema gababérgico. En efecto, la administración de fármacos que antagonizan la acción de GABA en el hipotálamo posterior inducen respuestas periféricas asociadas a estados de activación: incrementos en tasa cardiaca y respiratoria, y en presión sanguínea (DiMicco y colbs., 1986). Esta región de influencia sobre el sistema cardiovascular media, además, efectos comportamentales asociados a la ansiedad. Así, la inyección directa de muscimol (fármaco agonista GABA-A) produce una elevación en el número de respuestas castigadas en el paradigma de conflicto, lo que se interpreta como signo evidente de ansiolisis; por el contrario, bicuculina y picrotoxina (fármacos antagonistas GABA-A) reducen el número de dichas respuestas, sugiriendo ahora un efecto ansiógeno (Shekhar y colbs., 1990).

A conclusiones similares se ha llegado al inyectar diversas sustancias benzodiacepínicas en los cuerpos mamilares, estructuras anatómicas adyacentes al hipotálamo posterior. En efecto, la administración central de estos fármacos produce una acción ansiolítica en el paradigma de conflicto, sin afectar en ningún caso a las respuestas exentas de castigo (Kataoka y colbs., 1982; Yamashita y colbs., 1989).

Se especula con la posibilidad, pues, de que la región hipotalámica descrita sea, asimismo, uno de los posibles lugares de acción de sustancias ansiolíticas como las benzodiacepinas, y ello por varias razones: esta zona cerebral es parte integrante del circuito de Papez, posee receptores gabaérgicos sensibles al diacepam y fármacos relacionados, y lo que es más importante, modula respuestas fisiológicas y comportamentales íntimamente asociadas a la ansiedad.

\section{CONCLUSIONES}

Hace más de medio siglo, Papez proponía un sustrato neurobiológico para la conducta emocional, enfatizando el posible papel desempeñado por estructuras nerviosas integradas en el sistema límbico (Kuhar, 1986). Con el paso del tiempo, estas estructuras han demostrado su inequívoca participación en el control de las emociones, en estados tanto normales como patológicos (Brodal, 1981; Temoshok y colbs., 1983). De esta forma, y merced al advenimiento de sofisticadas técnicas experimentales, ha sido posible la precisa delimitación de circuitos cerebrales que canalizan, por ejemplo, la respuesta de miedo de un organismo ante una situación amenazante (Gray, 1987), la conducta agresiva (Bandler, 1982) o los centros nerviosos implicados en el reforzamiento (Rolls, 1981). Estos estu- 


\section{4}

dios, de carácter eminentemente localizacionista, evitan, sin embargo, reincidir en la falacia «núcleo cerebral-conducta», enfatizando la importancia de sistemas y circuitos frente a centros aislados.

El siempre difícil estudio de la ansiedad se ha visto enormemente influido por los descubrimientos neurobiológicos comentados a lo largo del presente trabajo. Concepto vago y escurridizo desde su inclusión por Freud en el ámbito psiquiátrico bajo el nombre de «angustia» (Ayuso, 1988), adquiere hoy una dimensión distinta, donde componentes de índole cognitivo, comportamental y fisiológico contribuyen a la expresión de un estado interno de enorme valor adaptativo para las especies (el organismo adquiere la capacidad de evitar situaciones potencialmente peligrosas, o se prepara para enfrentarse a un medio ambiente cambiante y hostil) (Kidman, 1989). Este estado armonioso puede, bajo determinadas circunstancias, ejercer un efecto altamente perturbador sobre el normal desenvolvimiento del sujeto, desembocando de lleno en el terreno de la psicopatología. En este sentido, la ansiedad patológica puede manifestarse de múltiples maneras, desde la existencia de un estado general de ansiedad (ansiedad generalizada), hasta la necesidad de completar tediosos e interminables rituales motores (trastornos obsesivo-compulsivos). La incidencia de estas alteraciones de conducta es, por otro lado, muy elevada en la población general, lo que justifica ampliamente la atención recibida desde un enfoque clínico (Reich, 1986). Este entusiasmo investigador ha alcanzado, asimismo, a los estudios de carácter básico, como fácilmente se desprende de la lectura del presente trabajo. Las cuestiones fundamentales que han guiado las diversas investigaciones de índole psicobiológico y neurobiológico relacionadas con la ansiedad pueden resumirse en los siguientes puntos:

1. ¿Es posible reproducir en animales de experimentación ese estado central definido como ansiedad?

2. ¿Qué estructuras cerebrales podrían estar mediatizando y/o modulando dicho estado central?

3. ¿A través de qué sistemas neuroquímicos y de qué forma actúan las sustancias ansiolíticas?

Un análisis detallado de la primera cuestión indica que los modelos animales de ansiedad gozan de buena reputación, en el sentido de poseer un buen valor predictivo (Treit, 1985). De hecho, las sustancias ansiolíticas son sistemáticamente estudiadas bajo la óptica de estos modelos como paso previo a su prescripción en humanos. Sustancias como las benzodiacepinas, los barbitúricos y el etanol inducen patrones comportamentales muy similares al ser administrados a sujetos experimentales: incrementan el número de respuestas castigadas en un paradigma de conflicto, incrementan también el número de cruces efectuados entre un compartimento iluminado y otro en penumbra, elevan el número de interacciones sociales, etc. Esto es: parecen decrementar el estado de «arousal» del sujeto (sin producir sedación) y desinhibir el comportamiento (Gray, 1987).

La segunda cuestión ha sido abordada en detalle en la presente revisión. Es obvio que las estructuras del sistema límbico, y sus intrincadas conexiones, son decisivas en el contexto de la ansiedad (Brodal, 1981; Kuhar, 1986), si bien es preciso incluir asimismo vías nerviosas de origen troncoencefálico (Gray, 1982a). Estos circuitos, en su totalidad, podrían armonizar el estado central de ansiedad y canalizar su expresión externa (a través de la conducta) e interna (a través del sistema nervioso autónomo y del sistema endocrino). 


\section{5}

Finalmente, y en tercer lugar, son varios los sistemas neuroquímicos implicados en la ansiedad, destacando los de GABA, NE y 5-HT. De todos ellos, el primero es el que mayor atención ha recibido, en especial el complejo receptor GABA-A, que incluye, junto a un lugar específico de unión para el propio GABA, otros tantos para las benzodiacepinas y los barbitúricos (Stephenson, 1988). Ello ha planteado la interesante posibilidad de la existencia de sustancias endógenas que modulen la acción celular de dicho complejo-receptor, culminando con el descubrimiento y caracterización de diversos endomoduladores de distinta naturaleza química (neuropéptidos y algunos metabolitos de hormonas esteroides), y estrechamente relacionados con procesos conductuales similares a la ansiedad (Morales, en trámite de publicación).

En conclusión, los estudios psicobiológicos y neurobiológicos realizados hasta la presente fecha sugieren de forma inequívoca que la ansiedad, constructo interno de características muy peculiares, se relaciona estrechamente con la actividad de determinados circuitos cerebrales y vías neuroquímicas asociadas, poseyendo, por consiguiente, un claro sustrato biológico al igual que otras conductas de naturaleza emocional. Es evidente que la explicación global de la ansiedad, tanto normal como patológica, no puede realizarse sólo desde una óptica biológica, ya que, como han demostrado los estudios psicológicos, posee además claros y bien diferenciados componentes cognitivos y conductuales, cuya descripción y análisis escapan a los objetivos del trabajo aquí desarrollado.

\section{Referencias}

Aghajanian, G. K.; Sprouse, J. S., y Rasmussen, K. (1977). Physiology of the midbrain serotonin system. En H. Y. Meltzer (Comp.): Psychopharmacology. The third generation of progress, 141-149. Nueva York: Raven Press.

American PsychiatricAssociation (1984). Manual Diagnóstico y Estadístico de los Trastomos Mentales. DSM-III. Barcelona: Masson.

Ayuso, J. L. (1988). Trastomos de angustia. Barcelona: Martínez Roca.

BANDLER, R. (1982). Neural control of aggressive behavior. Trends in Neurosciences, 5 , 390-394.

BRODAL, A. (1981). Neurological Anatomy in relation to Clinical Medicine. New York: OUP.

Carli, M.; Prontera , C., Y Samanin, R. (1989). Evidence that central 5-hydroxytryptaminergic neurones are involved in the anxiolytic activity of buspirone. Br. J. Pharmacol, 96, 829-836.

Carpenter, M. B. (1978). Core text of Neuroanatomy. Baltimore: Williams \& Wilkins.

CARrobles, J. A. (1989). La ansiedad: naturaleza, incidencias y modelos explicativos. En J. A. Carrobles (Comp.): Análisis y Modificación de Conducta II, Vol. I, 187-207. Madrid: U.N.E.D.

Cinnpman, W. P.; Schroeder, H. R.; Geyer, G.; Bezier, M. A. B.; Fagor, C.; Poppen, J. L.; SOLOMON, H. C., Y Yokovlev, P. I. (1954). Physiological evidence concerning importance of the amygdaloid nuclear region in the integration of circulatory function and emotions in man. Science, 120, 949-950.

ChOpin, P., y BEILEY, M. (1987). Animal models of anxiety: the effects of compounds that modify 5-HT neurotransmission. Trends in Pharmacol. Sci., 8, 383-388.

Clare, A. W. (1985). Anxiolytics in society. En S. D. Iversen (Comp.): Phychopharmacology. Recent advances and future prospects, 65-74. Oxford: OUP.

COSTA, E. (1985). Benzodiazepine/GABA interactions: a model to investigate the neurobio- 


\section{6}

logy of anxiety. En A. Hussain Tuma y J. D. Maser (Comps.): Anxiety and the anxiety disorders, 27.52. London: LEA.

DAvis, M. (1989). The role of the amygdala and its efferent projections in fear and anxiety. En P. Tyrer (Comp.): Psychopharmacology of Anxiety. Oxford: OUP.

Di Micco, J. A.; Abshire, V. M.; Hankins, K. D.; Sample, R. H. B., y Wible, J. H. Jr. (1986). Microinjection of GABA antagonists into posterior hypothalamus elevates heart rates in anesthetized rats. Neuropharmacol., 25, 1.063-1.066.

Eison, A. S.; Eison, M. S.; Stanley, M., y Riblet, L. A. (1986). Serotonergic mechanisms in the behavioral effects of buspirone and gepirone. Pharmacol. Biochem. E Behav., 24, 701-707.

Eriksson, E. (1987). Brain neurotransmission in panic disorder. Acta Psychiat. Scand., 76 (suppl. 335), 31-37.

Flaherty, C. F.; Grigson, P. S.; Demetrikopoulos, M. S.; Weaver, M. S.; Krauss, K. L., $Y$ RowAN, G. A. (1990). Effect of serotonergic drugs on negative contrast in consummatory behavior. Pharmacol. Biochem. E Behav., 36, 799-806.

Fonnum, F. (1987). Biochemistry, anatomy and pharmacology of GABA neurons. En H. Y. Meltzer (Comp.): Psychopharmacology. The third generation of progress, 173-182. Nueva York: Raven Press.

Gallager, D. W. (1978). Benzodiazepines: potentiation of a GABA inhibitory response in the dorsal raphe nucleus. Eur. J. Pharmacol., 49, $133-143$.

GARDNER, C. R. (1986). Recent developments in 5HT-related pharmacology of animal models of anxiety. Pharmacol. Biochem. E Behav., 24, 1.479-1.485.

$\mathrm{G}(\mathrm{A}, \mathrm{K}$. L., Y W $\mathrm{WRD}, \mathrm{A}$. (1986). Buspirone. A preliminary review of its pharmacological properties and therapeutic efficacy as an anxiolitic. Drugs, 32, 114-129.

Gold, M. S.; Redmond, D. E., y Kleber, H. D. (1978a). Clonidine in opiate withdrawal. Lancet, 1: 929-930.

Gold, M. S.; Redmond, D. E., y Kleber, H. D. (1987b). Clonidine blocks acute opiatewithdrawal symptoms. Lancet, 2, 599-602.

GraY, J. A. (1977). Drug effects on fear and frustration: possible limbic site of action of minor tranquillizers. En L. L. Iversen, S. D. Iversen y S. H. Snyder (Comps.): Handbook of Psychopharmacoly. Vol. VIII, 433-529. Nueva York: Plenum Press.

$\mathrm{G}_{\mathrm{RAY}}$ J. A. (1982a). The neuropsychology of anxiety: an enquiry into the functions of the septo bippocampal system. Oxford: OUP.

Gray, J. A. (1982b). Précis of the neuropsychology of anxiety: an enquiry into the functions of the septo-hippocampal system. Behav. Brain Sci., 5, 469-534.

Gray, J. A. (1987). The Psychology of fear and Stress (segunda edición). Cambridge: OUP.

HJORTH, S., y CARLSSON, A. (1982). Buspirone: effects on central monoaminergic transmission. Possicble relevance to animal experimentation and clinical findings. Eur. J. Pharmacol., 83, 299-303.

IVERSEN, S. D. (1985). Where in the central nervous system do benzodiazepines act? En S D. Iversen (Comp.): Psychopharmacology. Recent advances and future prospects, 75-88. Oxford: OUP

Joinston, A. L., y FILE, S. E. (1986). 5-HT and anxiety: Promises and pitfalls. Pharmacol. Biochem. E Behav., 24, 1.467-1.470.

Kahn, R. S.; Van Praag, H. M.; Wetzler, S.; Asnis, G. M., y Barr, G. (1988). Serotonin and anxiety revisited. Biol. Psychiat., 23, 189-208

Kataoka, Y.; Shibata, K.; Gomita, Y., y Veki, S. (1982). The mammillary body is a potential site of antianxiety action of benzodizepines. Brain Res., 241, 374-377.

Kataoka, Y.; Shibata, K.; Yamashita, K., y Veki, S. (1987). Differential mechanism involved in the anticonflict action of benzodiazepines injected into the central amygdala and mammillary body. Brin Res., 416, 243-247.

Kidman, A. (1989). Neurochemical and cognitive aspects of anxiety disorders. Prog. Neurobiol., 32, 391-402.

Kostowski, W.; Plaznik, A., y Stefanski, R. (1989). Intrahippocampal buspirone in animal models of anxiety. Eur. J. Pharmacol., 168, 393-396.

Kuhar, M. J. (1986). Neuroanatomical substrates of anxiety: A brief survey. Trends in Neu rosciences, $9,307-311$.

Lader, M., y Bruce, M. (1986). States of anxiety and their induction by drugs. Br. J. Clin. Pharmacol., 22, 251-261.

Lopes da Silva, F. H.; Witler, M. P.; Boeijinga, P. H., y Lehrman, A. H. M. (1990). Anatomic organization and physiology of the limbic cortex. Pbysiol. Rev., 70, 453-511.

Meldrun, B. S. (1990). Anatomy, physiology and pathology of epilepsy, Lancet, 336, 231-234.

Moore, R. Y. (1982). Catecholamine neuron systems in brain. Annals Neurol., 12, 321-327.

Morales, A. (en trámite de publicación). Principales sustancias endógenas implicadas en la modulación de la ansiedad.

Nauta, W. J. H., y FeirTaG, M. (1987). Fundamentos de Neuroanatomía. Barcelona: Lábor.

Nicoll, R. A.; Madison, D. V., y Lancaster, B. (1987). Noradrenergic modulation of neuronal excitability in mamalian hippocampus. En H. Y. Meltzer (Comp.): Psychopharmacology. The third generation of progress, 105-112. Nueva York: Raven Press. 
NienofF, D. L., y Kuhar, M. J. (1983). Benzodizepine receptors: localization in rat amygadala. J. Neurosci., 3, 2.091-2.097.

NisIIKAWA, T., Y SCATTON, B. (1986). Neuroanatomical site of the inhibitory influence of anxiolitic drugs on central serotonergic transmission. Brain Res., 371, 123-132.

O'CONNOR, J. J.; RowAN, M. J., y ANWYL, R. (1990). Actions of 5-HT 1 la ligands on excitatory synaptic transmission in the hippocampus of alert rats. Br. J. Pharmacol., 101, 171-177.

PASCOE, J. P., Y KAPP, B. S. (1985). Electrophysiological characteristics of amygdaloid central nucleus neurons during Pavlovian fear conditioning in rabbit. Behav. Brain Res., 16, $117-133$.

PelLow, S., y Filf, S. E. (1986). Anxiolytic and anxiogenic drug effects on exploratory activity in an elevated plus-maze: a novel test of anxiety in the rat. Pharmacol. Biochem. $\mathcal{E}$ Behav., 24, 525-529.

Rl:DMOND, D. E. (1987). Studies of the nucleus locus coeruleus in monkeys and hypotheses for neuropsychopharmacology. En H. Y. Meltzer (Comp.): Psychopharmacology. The third generation of progress, 967-976. Nueva York: Raven Press.

ReIC.H, J. (1986). The epidemiology on anxiety. J. Nerv. Ment. Dis, 174, 129-136.

Reiman, E. M.; Fusselman, M. J.; Fox, P. T., y Raichle, M. E. (1989). Neuroanatomical correlates of anticipatory anxiety. Science, 243, 1.071-1.074.

Reiman, E. M.; Raichle, M. E.; Butler, M. E.; Herscovitch, F. K., y Robins, E. (1984). A local brain abnormality in panic disorder, a severe form of anxiety. Nature, 310, 683-685.

RoLLs, E. T. (1981). Cerebro y recompensa. Barcelona: Fontanella.

Rowan, M. J.; Cullen, W. K., Y Moutton, B. (1990). Buspirone impairment of performance of passive avoidance and spatial learning tasks in the rat. Psychopharmacology, 100, 393-398.

SARTER, M., Y MARKOWITSCH, H. J. (1985). Involvement of the amygdala in learning and memory: A critical review, with emphasis on anatomical relations. Behav. Neurosci., 99, 342-380.

Scheel.Krüger, J., y Petersen, E. (1982). Anticonflict effect of the benzodiazepines mediated by a Gabaergic mechanism in the amygdala. Eur. J. Pharmacol., 82, 115-116.

SeGAL, M. (1980). The noradrenergic innervation of the hippocampus. En J. A. Hobson y M. A. B. Brazier (Comps.): The reticular formation revisited, 415-425. Nueva York: Raven Press.

Silekhar, A.; Hingtgen, J. N., y Dimicco, J. A. (1990). GABA receptors in the posterior hypothalamus regulate experimental anxiety in rats. Brain Res., 512, 81-88.

SIIIBATA, K.; KATAOKA, Y.; GoMITA, Y., Y VEKI, S. (1982). Localization of the site of the anticonflict action of benzodiazepines in the amygdaloid nucleus of rats. Brain Res., 234, 442-446.

Smiti, M. C.; Whisler, W. W., y Morrell, F. (1989). Neurosurgery of epilepsy. Seminars in Neurology, 9, 231-248.

Steinbusch, H. W. M., y Nieuweunnys, R. (1983). The raphe nuclei of the rat brainstem: a cytoarchitectonic and immunohistochemical study. En P. C. Emson (Comp.): Chemical Neuroanatomy, 131-207. Nueva York: Raven Press.

Stephenson, F. A. (1988). Understanding the GABA^ receptor: a chemically gated ion channel. Biochem. J., 249, 21-32.

SVENSSON, T. H. (1987). Peripheral, autonomic regulation of locus coeruleus noradrenergic neurons in brain: putative implications for psychiatry and psychopharmacology. Psychopbarmacology, 92, 1-7.

Taylor, D. P.; Eison, M. S.; Riblet, L. A., y Vandermaelen, C. P. (1985). Pharmacological and clinical effects of buspirone. Pharmacol. Biochem. \& Bebav., 23, 687-694.

Temoshor, L.; VAN Dike, C., Y Zegans, L. S. (Comps.) (1983). Emotions in health and illeness. Nueva York: Grune \& Stratto.

Tніевот, M. H. (1986). Are serotonergic neurons involved in the control of anxiety and in the anxiolitic activity of benzodiazepines? Pharmacol. Biochem. E Behav, 24, 1.471-1.477.

Thompson, R. F.; Berger, T. W.; BerRY, S. D.; Hoenler, F. K.; KetTNer, R. E., y Weisz, D. J. (1980). Hippocampal substrate of classical conditioning. Physiol. Psychol., 8, 262-279.

Traber, J., y Glaser, T. (1987). 5-HT IA receptor-related anxiolytics. Trends in Pharmacol. Sci., $8,432-437$.

TREIT, D. (1985). Animal models for the study of anti-anxiety agents: a reivew. Neurosci. Biobehav. Rev., 9, 203-222.

Trimble, M. R. (Comp.) (1986). New brain imagin techniques and psychopharmacology. Oxford: OUP.

Vandermaelen, C. P.; Matheson, G. K.; Wilderman, R. C., y Patterson, L. A. (1986). Inhibition of serotonergic dorsal raphe neurons by systemic and iontophoretic administration of buspirone, a non-benzodiazepine anxiolytic drug. Eur. J. Pharmacol., 129, 123-130.

VinogRadova, O. (1975). Functional organization of the limbic system in the process of registration of information: facts and hypotheses. En R. L. Isaacson y K. H. Pribam (Comps.): The Hippocampus. Vol. 2, 1-70. Nueva York: Plenum Press.

Yamasiita, K.; Kataoka, Y.; Miyazaki, A.; Shibata, K.; Tominaga, K., y Veki, S.(1989). A key role of the mammillary body in mediation of the antianxiety action of zopiclone, a cyclopyrrolone derivate. Japan J. Pharmacol., 51, 438-442. 